\title{
Left Anterior Prefrontal Activation Increases with Demands to Recall Specific Perceptual Information
}

\author{
Charan Ranganath, ${ }^{1}$ Marcia K. Johnson, ${ }^{2}$ and Mark D'Esposito ${ }^{1}$ \\ ${ }^{1}$ Helen Wills Neuroscience Institute and Department of Psychology University of California, Berkeley, California, and \\ 2Department of Psychology, Yale University, New Haven, Connecticut
}

Results from neuroimaging studies have led to competing theories regarding the contributions of prefrontal regions to memory formation and retrieval. To investigate this issue, we used event-related functional magnetic resonance imaging to assess prefrontal activation during encoding and retrieval of pictures of objects. Responses to studied and unstudied objects at retrieval were compared between two tests with differing demands for the specificity of information to be retrieved (source vs old-new recognition). Results showed that bilateral ventral [Brodmann's areas (BA) 44, 45, and 47] and right dorsal (BA 9) prefrontal regions were activated during both encoding and retrieval, but activity in these regions was not reliably modulated by the specificity of information to be retrieved. A region in left anterior prefrontal cortex (BA 10/46) was reliably activated during retrieval trials, and activation in this region increased with demands to retrieve perceptually detailed information about studied objects. Our results show that left anterior prefrontal cortex is engaged during the monitoring and evaluation of specific memory characteristics at retrieval-a process critical for accurate episodic remembering.

Key words: prefrontal cortex; frontal lobes; episodic memory; source monitoring; event-related fMRI; neuroimaging
It has long been known that prefrontal cortex (PFC) contributes to long-term memory, and recent neuroimaging results have supported the hypothesis that different prefrontal regions implement different functions (Buckner and Petersen, 1996; Nyberg et al., 1996; Nolde et al., 1998a; Wagner, 1999). For example, Wagner (1999) suggested that left and right ventral prefrontal regions [Brodmann's areas (BA) 44, 45, and 47] implement working memory maintenance operations during encoding and retrieval, whereas right dorsal (BAs 9 and 46) and anterior (BA 10) prefrontal regions implement monitoring and manipulation processes engaged primarily at retrieval. In particular, the idea that right anterior prefrontal cortex is engaged specifically during episodic retrieval is consistent with neuroimaging results showing that this region is particularly engaged during episodic retrieval tasks (Buckner and Petersen, 1996; Nyberg et al., 1996).

However, a closer examination of available evidence suggests that laterality within dorsal and anterior PFC may be more complex than previously suggested. In their review of the neuroimaging literature, Nolde et al. (1998a) observed that relatively simple memory retrieval tasks elicited right prefrontal activation, but more demanding retrieval tasks elicited left prefrontal activation as well. Consistent with this observation, subsequent studies investigating the kinds of source memory tests that are sensitive to prefrontal damage (Johnson et al., 1993; Shimamura, 1995) revealed left-lateralized or bilateral activation in dorsal and anterior PFC (Nolde et al., 1998b; Henson et al., 1999; Rugg et al., 1999; Raye et al., 2000).

Using a source memory paradigm introduced in previous eventrelated potential (ERP) studies of memory retrieval (Ranganath and Paller, 1999, 2000), we sought to specify the roles of left and

Received May 8, 2000; revised Aug. 30, 2000; accepted Aug. 30, 2000.

This research was supported by grants from the American Federation for Aging Research (M.D.), the McDonnell-Pew Program in Cognitive Neuroscience (C.R.), and National Institute on Aging Grants AG05863, AG15793, and AG09253.We thank Jeff Berger, Dan Caggiano, and Alexander Taich for technical support, and Mick Rugg and Rik Henson for comments on a previous version of this manuscript. Special thanks to Ken A. Paller for his collaboration on the ERP studies that inspired this work.

Correspondence should be addressed to Dr. Charan Ranganath, University of California, Department of Psychology, 3210 Tolman Hall, \#1650, Berkeley, CA 94720-1650. E-mail: charan@socrates.berkeley.edu.

Copyright (C) 2000 Society for Neuroscience 0270-6474/00/200001-05\$15.00/0 right dorsal, ventral, and anterior prefrontal regions during episodic remembering using rapid presentation event-related functional magnetic resonance imaging (fMRI). We first examined activity in these prefrontal regions during both encoding and retrieval. Next, we identified whether activation of these regions during retrieval reflected the reactivation of stored information ("retrieval success") or the implementation of monitoring and evaluation processes (Johnson et al., 1993, 1997; Rugg et al., 1996).

Participants were scanned during encoding of objects and during retrieval tests that included size-changed versions of studied objects and unstudied objects (Fig. 1 $A$ ). During the "general" test, participants identified whether each object was old or new, whereas during the "specific" test, they additionally specified the direction of size change for studied objects. Because the specific test placed greater demands on the recall and evaluation of perceptual attributes of studied objects than did the general test, we were separately able to determine whether prefrontal activation at retrieval was related to: (1) reactivation of stored information (i.e., the contrast between old and new trials), and (2) monitoring and evaluation of specific memory characteristics at retrieval (i.e., the contrast between specific and general trials).

\section{MATERIALS AND METHODS}

Participants. Six males and two females from the University of Pennsylvania community were paid for participating in the experiment. All were right-handed and ranged in age from 18 to 28 years. Data from one additional participant were discarded because of excessive motion artifact. Stimuli. Stimuli were 240 grayscale photos and drawings of common

This article is published in The Journal of Neuroscience, Rapid Communications Section, which publishes brief, peer-reviewed papers online, not in print. Rapid Communications are posted online approximately one month earlier than they would appear if printed. They are listed in the Table of Contents of the next open issue of JNeurosci. Cite this article as: JNeurosci, 2000, 20:RC108 (1-5). The publication date is the date of posting online at www.jneurosci.org.

http://www.jneurosci.org/cgi/content/full/4722 


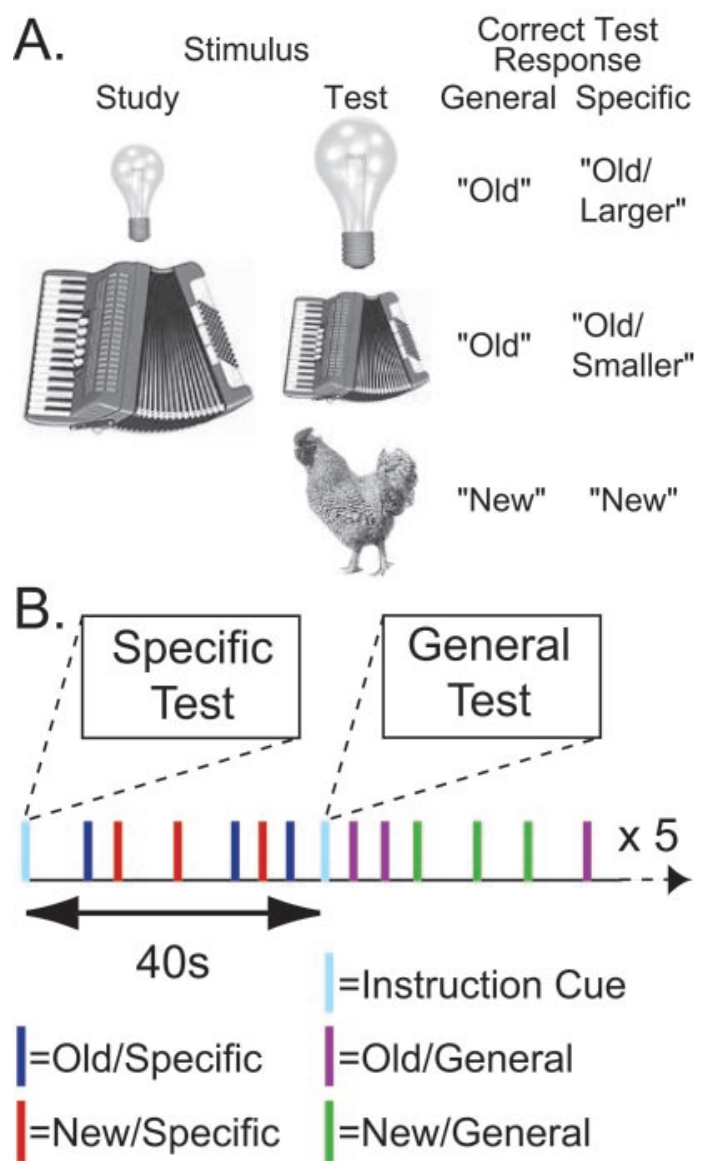

Figure 1. A, Example stimuli and responses for each test condition. During the test phase, participants saw size-changed versions of studied objects and unstudied objects. In the general (old-new recognition) test, instructions were to respond "Old" for all studied objects, regardless of the size change. In the specific (source) test, instructions were to indicate whether studied objects were shown in a larger or smaller size at test. "New" responses were identical across the two test conditions. B, A schematic depiction of the sequence of events during a test run is shown, with colored bars representing the onset of each event on a timeline. Test conditions were blocked, and each $40 \mathrm{sec}$ block began with an instruction cue indicating what test instructions to follow. Note that because the length of the intertrial interval (ITI) and the sequence of old and new trials within each block were randomized, it was possible to use eventrelated analysis techniques (see Materials and Methods).

objects ("normal size") and versions of these objects that were scaled to $65 \%$ ("reduced size"), and $155 \%$ ("enlarged size"). These stimuli were provided courtesy of Nancy Kanwisher (Massachusetts Institute of Technology, Cambridge, MA) and Michael J. Tarr, (Brown University, Providence, RI). To eliminate confounding material-specific effects, the mapping of objects to test (specific vs general) and item type (old vs new) was counterbalanced across subjects.

Behavioral procedure. Before scanning, participants practiced each task to assure that they fully understood the instructions. In the scanner, stimuli were presented on a backlit projection screen that subjects viewed through a mirror mounted on the head coil. Manual responses were made with a four-button fiber-optic response pad. The experiment consisted of alternating runs in which participants studied objects and were then tested on them.

In each of four study runs, 30 enlarged or reduced size objects were presented twice each. The trials were in a pseudorandom sequence such that novel and repeated encoding trials were intermixed. On each encoding trial, a picture was presented for $1 \mathrm{sec}$ and replaced by a fixation cross through the remainder of the intertrial interval (ITI). To allow for modeling of fMRI responses to each trial relative to baseline, the ITI was randomly jittered from 3 to $7 \mathrm{sec}$ in the study phase (Dale and Buckner, 1997; Josephs and Henson, 1999). During encoding, participants were to push the far right button if the object was judged to be taller than it was wide or to push the left button if the object was wider than it was tall. This orienting task was used to control encoding strategies such that each object was encoded in a perceptually detailed manner. On average, the delay between the last picture in the study phase and the beginning of the test phase was $\sim 2-3 \mathrm{~min}$. Across runs, there were 240 encoding trials (120 novel, 120 repeated).

After each study run, participants were tested on the 30 objects that they had just studied. They were instructed that they would see reduced (old-smaller) and enlarged (old-larger) versions of studied objects and previously unseen (new) objects. In actuality, only normal size pictures were presented during the test phase, but because participants studied only reduced and enlarged pictures, all old objects in the test phase were larger or smaller than the studied objects. During each test run, participants alternated between five blocks of each of two test conditions. Each block included three old and three new objects. For specific test blocks, instructions were to push the far left button for old-larger objects, the mid-left button for old-smaller objects, and the far right button for new objects. For general test blocks, instructions were to ignore size changes and push the mid-right button for old objects and the far right button for new objects. In both test conditions, participants made a single button response for each trial and responded to old objects with the left thumb and new objects with the right thumb. Thus, in the specific test, participants used the left thumb to press either of the two buttons corresponding to old-larger and old-smaller responses.

At the beginning of each test block, an instruction cue (indicating which test was to be performed) was presented for $1 \mathrm{sec}$ and was then replaced by a fixation cross for the next $3 \mathrm{sec}$, followed by a pseudorandom sequence of six test trials. On each test trial, an old-larger, oldsmaller, or new object was presented for $1 \mathrm{sec}$, followed by a fixation cross through the remainder of the ITI, which varied from 3 to $11 \mathrm{sec}$. Across runs, there were 60 trials for each combination of test condition (specific vs general) and item type (old vs new).

fMRI procedure. Functional images sensitive to blood oxygenation level-dependent (BOLD) contrast were acquired using a gradient echo echoplanar sequence (repetition time, $2000 \mathrm{msec}$; echo time, $50 \mathrm{msec}$; matrix size, $64 \times 64$; field of view, $24 \mathrm{~cm}$ ). Each functional volume consisted of 21 contiguous 5-mm-thick axial slices. Preprocessing of functional images included: (1) sinc interpolation in time to correct for between-slice timing differences in image acquisition, (2) motion correction using a six-parameter, rigid-body transformation algorithm provided by Statistical Parametric Mapping (SPM96) software, (3) using a partial correlation method, and (4) normalization of the time series of each voxel by its mean signal value to attenuate between-run scaling differences.

fMRI analysis methods. All analyses were performed within the framework of the modified general linear model (GLM) of Worsley and Friston (1995), incorporating empirically derived estimates of temporal autocorrelation (Zarahn et al., 1997). The design matrix for each analysis included covariates representing task-related variables and filters to remove frequencies $>0.25 \mathrm{~Hz}$ and $<0.01 \mathrm{~Hz}$.

A hemodynamic response function (HRF) was empirically derived for each subject based on results from a visuomotor response task that was performed before the experimental tasks (Aguirre et al., 1998). BOLD responses to study and test trials were modeled by HRFs coinciding with the onset of each event, using fixation images acquired during the ITI as a baseline. Parameter estimates (measuring response amplitude) yielded by the GLM for each event type were used to compute $t$ statistics. Separate statistical parametric maps (SPMs) of $t$ values for contrasts of interest were made for each subject. These SPMs were spatially normalized to the template from the International Consortium for Brain Mapping Project and resliced into 5-mm-thick axial slices using algorithms from SPM96 software. This stereotaxic space approximates, but is not identical to the coordinate system defined by Talairach and Tournoux (1988).

Maps were then smoothed with a three-voxel-wide Gaussian filter to account for remaining between-subject anatomical variability. Finally, for each contrast of interest, a random-effects analysis was performed to test whether the mean of the individual subjects' $t$ values at each voxel was reliably different from zero. Because we were primarily interested in characterizing response properties of prefrontal regions previously implicated in imaging studies of episodic retrieval, we restricted our discussion to BAs 9, 10, 44, 45, 46, and 47. Random-effects SPMs were thresholded such that only clusters of six or more contiguous voxels exceeding $t_{(7)}>+3.50$ (corresponding to a one-tailed uncorrected $p<0.005$ ) were included. Results from critical comparisons were confirmed by comparing the magnitudes of parameter estimates yielded by the GLM for each condition of interest. All results were significant even when smaller smoothing kernels (or no smoothing) were applied to the data.

\section{RESULTS}

\section{Behavioral results}

Given that each object was encoded twice, reaction times (RTs) for shape judgments during the study phase were computed separately for the two exposures. Mean RTs were significantly faster during the second study exposure (mean, $845.0 \mathrm{msec}$; SD, 183.8) than during the first (mean, $961.3 \mathrm{msec}$; SD, 149.8) $\left(t_{(7)}=\right.$ 5.36; $p=0.001)$. 
Table 1. Prefrontal regions showing BOLD signal increases during encoding and/or retrieval trials relative to fixation

\begin{tabular}{|c|c|c|c|c|c|c|c|c|c|}
\hline \multirow[b]{2}{*}{ Region } & \multirow[b]{2}{*}{ BA } & \multicolumn{4}{|c|}{ Encoding } & \multicolumn{4}{|c|}{ Retrieval } \\
\hline & & $X$ & $Y$ & $Z$ & $t(7)$ & $X$ & $Y$ & $Z$ & $t(7)$ \\
\hline $\begin{array}{l}\text { R. anterior inferior } \\
\text { frontal gyrus }\end{array}$ & 47 & 26 & 23 & -10 & 6.21 & 23 & 30 & -10 & 4.39 \\
\hline $\begin{array}{l}\text { L. anterior inferior } \\
\text { frontal gyrus }\end{array}$ & 47 & -38 & 19 & 0 & 5.31 & $\begin{array}{l}-34 \\
-19 \\
-30\end{array}$ & $\begin{array}{r}19 \\
4 \\
26\end{array}$ & $\begin{array}{r}10 \\
-20 \\
-15\end{array}$ & $\begin{array}{r}11.13 \\
5.68 \\
3.67\end{array}$ \\
\hline $\begin{array}{l}\text { L. posterior infe- } \\
\text { rior frontal gyrus }\end{array}$ & $44 / 45$ & -41 & 23 & 20 & 4.09 & $\begin{array}{l}-53 \\
-56\end{array}$ & $\begin{array}{l}15 \\
34\end{array}$ & $\begin{array}{l}20 \\
10\end{array}$ & $\begin{array}{l}7.40 \\
6.31\end{array}$ \\
\hline $\begin{array}{l}\text { R. posterior infe- } \\
\text { rior frontal gyrus }\end{array}$ & $44 / 45$ & $\begin{array}{l}45 \\
23\end{array}$ & $\begin{array}{l}30 \\
30\end{array}$ & $\begin{array}{l}20 \\
25\end{array}$ & $\begin{array}{l}5.20 \\
4.16\end{array}$ & 45 & 30 & 25 & 7.89 \\
\hline $\begin{array}{l}\text { R. superior frontal } \\
\text { gyrus }\end{array}$ & 9 & 23 & 45 & 30 & 5.34 & 26 & 34 & 30 & 4.99 \\
\hline $\begin{array}{l}\text { L. middle frontal } \\
\text { gyrus }\end{array}$ & $9 / 46$ & -41 & 30 & 25 & 4.32 & & & & \\
\hline $\begin{array}{l}\text { L. anterior middle } \\
\text { frontal gyrus }\end{array}$ & $10 / 46$ & & & & & $\begin{array}{l}-53 \\
-34\end{array}$ & $\begin{array}{l}41 \\
41\end{array}$ & $\begin{array}{l}0 \\
0\end{array}$ & $\begin{array}{l}5.64 \\
5.14\end{array}$ \\
\hline
\end{tabular}

Note R, Right; L, left; BA, Brodmann's area.

Test phase behavioral data were computed for seven of the eight participants (because of technical difficulties, behavioral responses could not be recorded for one subject). Old-new recognition accuracy results, computed regardless of size judgment accuracy in the specific test, showed that participants were able to accurately identify old (specific test: mean, 93.8\%, SD, $4.8 \%$; general test: mean, 94.3\%, SD, 2.5\%) and new (specific test: mean, 95.3\%, SD, 2.2\%; general test: mean, 91.7\%, SD, 4.1\%) objects. An ANOVA on recognition accuracy with item type (old vs new) and test condition (specific vs general) revealed no reliable old-new accuracy differences between item types or test conditions (all $F_{(1,6)}$ values $<2.3$; all $p$ values $>0.15$ ). The mean accuracy of size judgments for old objects in the specific test was $88 \%$ (range, 78-93; SD, 6.5\%), which was significantly above chance $\left(t_{(6)}=14.47 ; p<0.001\right)$.

An ANOVA on reaction times during the test phase revealed that, overall, reaction times were slower in the specific than the general test $\left(F_{(1,6)}=31.56 ; p=0.001\right)$ and slower for old than for new items $\left(F_{(1,6)}=80.96 ; p<0.001\right)$, but these main effects were qualified by a significant item type $\times$ test condition interaction $\left(F_{(1,6)}=37.22 ; p=0.001\right)$. Follow-up analyses on the simple effects revealed that responses were slower to old objects in the specific test than in the general test (mean, $1573.2 \mathrm{msec}, \mathrm{SD}, 360.6$ and mean, 1039.37 msec, $\mathrm{SD}, 247.3$, respectively; $t_{(6)}=6.2 ; p=$ $0.001)$, but no reliable differences were observed in reaction times to new objects across the two test conditions (specific test: mean, 939.5 msec, SD, 212.0; general test: mean, $947.4 \mathrm{msec}, \mathrm{SD}, 203.4$, respectively; $\left.t_{(6)}<1\right)$.

\section{FMRI results: encoding phase}

Talaraich coordinates of local maxima of prefrontal activation during encoding trials are shown in Table 1. Results, shown in Figure $2 A$, revealed that regions in the left and right inferior frontal gyri (BA 44, 45, and 47), the right superior frontal frontal gyrus (BA 9), and the left dorsal middle frontal gyrus (BA 9/46) were activated during encoding trials. Given that several studies have reported decreases in prefrontal activation when an item is processed multiple times in the same manner (Buckner et al.,
1998), we explored whether any prefrontal regions showed reliable repetition-related signal changes during encoding. Although no prefrontal regions showed reliable reductions in activation with item repetition, a region in the left anterior middle frontal gyrus (BA 10/46), shown in Figure $2 B$, exhibited increased activation when objects were encoded for the second time relative to the first encoding $\left(x=-45 ; y=49 ; z=0 ; t_{(7)}=12.87\right.$; number of voxels, 103). Mean parameter estimates for activation in this region during the first and second encoding trials were 0.0001 (SE, 0.0002) and 0.0004 (SE, 0.0015), respectively.

\section{FMRI results: retrieval phase}

Talaraich coordinates of local maxima of prefrontal activation during retrieval trials are shown in Table 1. As shown in Figure $2 C$, regions in the left and right inferior frontal gyri (BA 44, 45, and 47), the right superior frontal frontal gyrus (BA 9), and the left anterior middle frontal gyrus (BA 10/46) were activated during retrieval trials relative to baseline.

The design of the present experiment allowed us to not only identify regions that showed reliable increases in activity during retrieval trials, but to additionally characterize how this activity varied according to retrieval demands. Results of the direct contrast between the two test conditions, shown in the top left panel of Figure 3, revealed that activation within the left anterior middle frontal gyrus (BA 10/46) was greater for specific than general test trials $\left(x=-45 ; y=49 ; z=5 ; t_{(7)}=4.88\right.$; number of voxels, 28). Further analyses, shown in the lower two rows of Figure 3, confirmed that this region was reliably activated for both old and new trials relative to the ITI during the specific test. No other prefrontal regions were reliably activated in this contrast.

We conducted a test condition $\times$ item type ANOVA on $t$ values associated with each trial type in the left prefrontal region identified in the specific-general contrast. As expected, this region showed greater activation for specific than general test trials $\left(F_{(1,7)}=22.23 ; p=0.002\right)$. In addition, the region exhibited marginally greater activation during old trials than new trials $\left(F_{(1,7)}=3.70 ; p=0.096\right)$, but no significant interaction was observed $\left(F_{(1,7)}<1\right)$. Virtually identical results were observed 


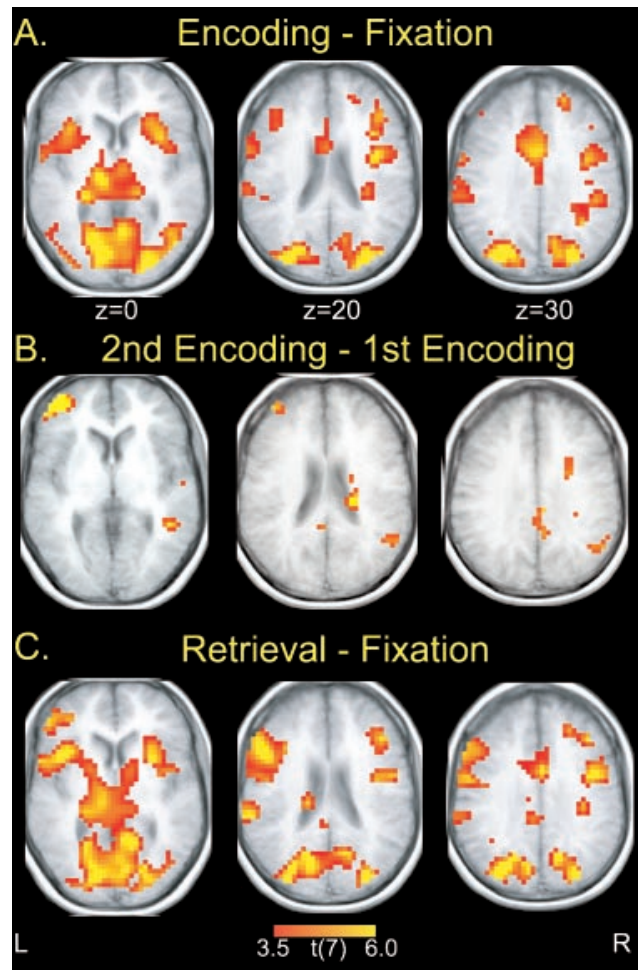

Figure 2. Activation during encoding and retrieval trials. A, Regions showing enhanced activation during encoding trials relative to the ITI are shown. Activations are overlaid on an average of the spatially normalized anatomical images from the eight participants. Left $=$ Left on these and all subsequent images. $B$, Results from the same slices are shown for the analysis of activation during repeated object encoding relative to the first time each object was encoded. $C$, Results from the same slices are shown for the analysis of activation during retrieval trials relative to the ITI. These images reveal that bilateral regions in anterior and posterior inferior frontal gyri (BAs 44, 45, and 47) and the right superior frontal gyrus (BA 9) were active during both encoding and retrieval trials. In addition, a region in the left anterior middle frontal gyrus (BA 10/46) was active during retrieval trials and showed more activation during the second time each object was encoded relative to the first.

when the analysis was repeated on measurements of response amplitudes, shown in the top right panel of Figure 3. These findings demonstrate that left anterior PFC was more activated during specific than general test trials for new as well as old items.

Mapwise contrasts between old and new trials revealed two regions of activation within prefrontal cortex. A small region in the posterior portion of the left middle frontal gyrus (BA 9) showed enhanced responses during old trials $(x=-45 ; y=19$; $z=30 ; t_{(7)}=4.53$; number of voxels, 15$)$, and a small region in right anterior inferior frontal gyrus (BA 47) showed enhanced responses to new objects $\left(x=38 ; y=-34 ; z=-10 ; t_{(7)}=4.44\right.$; number of voxels, 8$)$. These regions were not activated in the specific-general contrast and therefore appear to be differentially activated as a result of previous exposure, regardless of the specificity of retrieval demands.

\section{DISCUSSION}

In the present study we investigated how different prefrontal regions contribute to episodic memory. By using rapidpresentation event-related fMRI within the context of a blocked task paradigm, we were able to identify the extent to which prefrontal activity at retrieval was modulated by the specificity of source information to be retrieved. Results showed that a region within the left anterior middle frontal gyrus (BA 10/46) was activated during retrieval trials and that activation within this

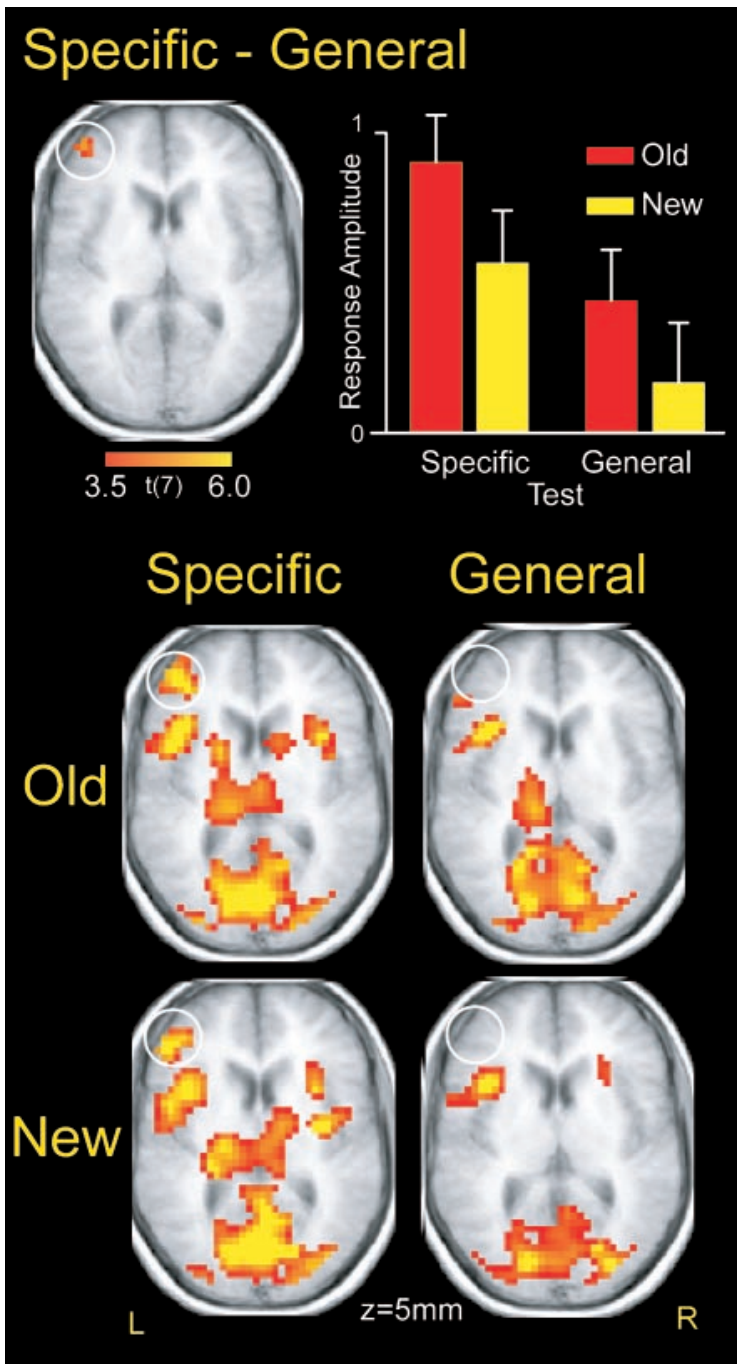

Figure 3. Left anterior prefrontal cortex was more active for specific than general test trials. Results from the contrast between specific and general test trials are shown in the top left panel of the figure. At bottom, results from the same slice are shown for the analysis of old and new items relative to fixation separately for each test condition. Note that the circled region in the left anterior middle frontal gyrus (BA 10/46) was more active for specific than the general test trials. At the top right panel of the figure, a bar graph shows parameter estimates, measuring response amplitudes for each trial type relative to the ITI. Error bars show the SEM across participants. These results confirm that left anterior PFC was more active during specific than during general trials and that similar modulations were observed for both old and new objects.

region was enhanced during specific (size judgment) relative to general (old-new recognition) trials.

Results from previous studies suggest that left PFC is recruited during memory tasks that demand specific source attributions (Nolde et al., 1998b; Ranganath and Paller, 1999; Rugg et al., 1999; Ranganath and Paller, 2000; Raye et al., 2000), and the present results allow us to further specify the role of left anterior PFC in episodic memory. In light of the fact that this region was activated during both old and new trials in the specific test, it is unlikely that left anterior PFC activation solely reflects retrieval success. Instead, our results suggest that this region implements monitoring or evaluative processes that are engaged when one attempts to retrieve information from memory, and that such processes are applied to old and new items. Indeed, according to the source-monitoring framework, such monitoring processes are applied to old and new items to differentiate between them (Johnson et al., 1993). 
We also observed that left anterior PFC exhibited greater activation during the second time each object was encoded relative to the first. This region also exhibited marginally greater activation during old than new trials at retrieval. Consistent with these findings, an almost identical region showed increased activation during recognition hits relative to correct rejections in another recent event-related fMRI study (Konishi et al., 2000). These findings suggest that left anterior PFC activation can be modulated by previous exposure to an item. It is reasonable to assume that when confronted by a repeated object, a feeling of familiarity induces additional source monitoring processing (Johnson and Raye, 1981; Johnson et al., 1993). Accordingly, the fact that left anterior prefrontal activation was enhanced after repeated object processing is consistent with our proposal that this region mediates the selection and evaluation of specific memory characteristics.

An alternate explanation for the difference in activation between specific and general trials in left anterior PFC at retrieval is that this was secondary to differences in accuracy and/or reaction times between the two test conditions. But although accuracy and RTs for old objects differed between the two test conditions, behavioral results for new objects were similar across the two tests. Nonetheless, left anterior PFC responses during both old and new trials were greater during the specific than the general test (Fig. 3). Thus, it is unlikely that differential activation between the test conditions can be attributed to performance differences.

This conclusion is bolstered by electrophysiological results showing temporal dynamics of left frontal brain activity during similar specific and general tests (Ranganath and Paller, 1999, 2000). For example, results from one ERP study (Ranganath and Paller, 1999) showed that ERP differences between specific and general tests were apparent at left frontal scalp sites as early as $200 \mathrm{msec}$ after stimulus onset and persisted throughout the 1200 msec recording epoch. Furthermore, these ERP differences were seen for both old and new objects, despite the fact that RTs and accuracy for new objects did not differ between the two test conditions. Based on the temporal dynamics of frontal activity observed in these ERP studies, we hypothesize that left anterior PFC was critical for the selection and evaluation of specific memory characteristics (Johnson and Raye, 1981; Johnson et al., 1993).

In contrast to our findings of robust left anterior prefrontal activation, we did not observe reliable activation in right anterior PFC (BA 10), a region frequently activated in neuroimaging studies of episodic memory retrieval (Buckner and Petersen, 1996; Nyberg et al., 1996; Nolde et al., 1998a; Wagner, 1999). It is unclear whether this null result was attributable to insufficient statistical power or caused by differences in task design between the present study and other studies that observed right anterior PFC activation. Importantly, although this region is frequently activated during simple retrieval tasks, it is clearly less likely than left anterior PFC to exhibit increased activation during tasks that require more specific memory attributions (Nolde et al., 1998b; Henson et al., 1999; Ranganath and Paller, 1999; Rugg et al., 1999; Ranganath and Paller, 2000; Raye et al., 2000).

Findings from previous neuroimaging studies have led to the suggestion that ventral prefrontal regions (BAs 44, 45, and 47) implement working memory operations at both encoding and retrieval, whereas dorsal prefrontal regions (BAs 9 and 46) are engaged primarily during retrieval (Wagner, 1999). Consistent with this view, our results showed that bilateral inferior frontal gyri (BAs 44, 45, and 47) were activated at both encoding and retrieval. But we also found that a region in the left middle frontal gyrus (BA 9/46) was activated during encoding, and another in the right superior frontal gyrus (BA 9) was activated during both encoding and retrieval trials. These findings suggest that, although dorsal and ventral prefrontal regions may implement distinct reflective processes, these component processes can be flexibly engaged by both encoding and retrieval tasks (Johnson et al., 1993; D’Esposito et al., 2000).

In conclusion, the present results add to accumulating evidence that prefrontal cortex is not a functionally homogeneous region (Buckner and Petersen, 1996; Wagner, 1999; D'Esposito et al., 1998, 2000; Raye et al., 2000). Left and right prefrontal regions contributed to performance on the memory tasks in this study, but a region within left anterior prefrontal cortex was especially engaged during the evaluation of specific memory characteristics. Given evidence that this process is critical for specifying the source of a memory (Johnson et al., 1993), including differentiating these memories from thoughts or imagined events (Johnson and Raye, 1981), our findings underscore the importance of this region for accurate recollection.

\section{REFERENCES}

Aguirre GK, Zarahn E, D'Esposito M (1998) The variability of human, BOLD hemodynamic responses. NeuroImage 8:360-369.

Buckner RL, Petersen SE (1996) What does neuroimaging tell us about the role of prefrontal cortex in memory retrieval? Semin Neurosci 8:47-55.

Buckner RL, Goodman J, Burock M, Rotte M, Koutstaal W, Schacter D, Rosen B, Dale AM (1998) Functional-anatomic correlates of object priming in humans revealed by rapid presentation event-related fMRI. Neuron 20:285-296.

Dale AM, Buckner RL (1997) Selective averaging of rapidly presented individual trials using fMRI. Hum Brain Mapp 5:1-12.

D'Esposito, Aguirre GK, Zarahn E, Ballard D (1998) Functional MRI studies of spatial and non-spatial working memory. Cognitive Brain Res 7:1-13.

D'Esposito M, Postle BR, Rypma B (2000) Prefrontal cortical contributions to working memory: evidence from event-related fMRI studies. Exp Brain Res 133:3-11.

Henson RNA, Shallice T, Dolan RJ (1999) Right prefrontal cortex and episodic memory retrieval: a functional MRI test of the monitoring hypothesis. Brain 122:1367-1381.

Johnson MK, Raye CL (1981) Reality monitoring. Psychol Rev 88:67-85.

Johnson MK, Hashtroudi S, Lindsay DS (1993) Source monitoring. Psychol Bull 114:3-28.

Johnson MK, Kounios J, Nolde SF (1997) Electrophysiological brain activity and memory source monitoring. NeuroReport 8:1317-1320.

Josephs O, Henson RN (1999) Event-related functional magnetic resonance imaging: modelling, inference and optimization. Philos Trans R Soc Lond B Biol Sci 354:1215-1228.

Konishi S, Wheeler ME, Donaldson DI, Buckner RL (2000) Neural correlates of episodic retrieval success. NeuroImage 12:276-286.

Nolde SF, Johnson MK, Raye CL (1998a) The role of prefrontal regions during tests of episodic memory. Trends Cognit Sci 2:399-406.

Nolde SF, Johnson MK, D'Esposito M (1998b) Left prefrontal activation during episodic remembering: an event-related fMRI study. NeuroReport 9:3509-3514.

Nyberg L, Cabeza R, Tulving E (1996) PET studies of encoding and retrieval: the HERA model. Psychon Bull Rev 3:135-148.

Ranganath C, Paller KA (1999) Frontal brain potentials during recognition are modulated by requirements to retrieve perceptual detail. Neuron 22:605-613.

Ranganath C, Paller KA (2000) Neural correlates of memory retrieval and evaluation. Brain Res Cogn Brain Res 9:209-222.

Raye CL, Johnson MK, Mitchell KJ, Nolde SF, D’Esposito M (2000) fMRI investigations of left and right PFC contributions to episodic remembering. Psychobiology 28:197-206.

Rugg MD, Fletcher PC, Frith CD, Frackowiak RSJ, Dolan RJ (1996) Differential activation of the prefrontal cortex in successful and unsuccessful memory retrieval. Brain 119:2073-2083.

Rugg MD, Fletcher PC, Chua PM, Dolan RJ (1999) The role of the prefrontal cortex in recognition memory and memory for source: an fMRI study. NeuroImage 10:520-529.

Shimamura AP (1995) Memory and frontal lobe function. In: The cognitive neurosciences (Gazzaniga MS, ed), pp 803-813. Cambridge, MA: MIT.

Talairach J, Tournoux P (1988) Co-planar stereotaxic atlas of the human brain. New York: Thieme.

Wagner AD (1999) Working memory contributions to human learning and remembering. Neuron 22:19-22.

Worsley KJ, Friston KJ (1995) Analysis of fMRI time-series revisitedagain. NeuroImage 2:173-182.

Zarahn E, Aguirre GK, D'Esposito M (1997) Empirical analyses of BOLD fMRI statistics. I. Spatially unsmoothed data collected under null-hypothesis conditions. NeuroImage 5:179-197. 